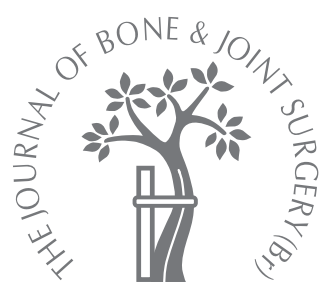

J. E. Robb

From the Royal

Hospital for Sick

Children, Edinburgh, Scotland

J. E. Robb, MD, FRCS, FRCP,

Consultant Orthopaedic

Surgeon

Royal Hospital for Sick

Children, Sciennes Road,

Edinburgh EH9 1LF, Scotland.

Correspondence should be sent

to $\mathrm{Mr}$ J. E. Robb; e-mail:

j.e.robb@btinternet.com

(C)2009 British Editorial Society

of Bone and Joint Surgery

doi:10.1302/0301-620X.91B11.

$23349 \$ 2.00$

$J$ Bone Joint Surg $[\mathrm{Br}]$ 2009;91-B:1410-12.

- ANNOTATION

\title{
The pink, pulseless hand after supracondylar fracture of the humerus in children
}

\begin{abstract}
This annotation discusses the findings of two papers in the current issue describing the management of the neurovascular complications of supracondylar fractures of the humerus in childhood, with particular reference to the indications for and the timing of exploration of the brachial artery and the affected nerves.
\end{abstract}

Extension supracondylar fractures of the humerus are the most common fractures involving the elbow in children and the literature reports approximately a $10 \%$ to $20 \%$ incidence $^{1}$ of an absent radial pulse in children presenting with a Gartland ${ }^{2}$ type III fracture. This is not an emergency provided that the hand is well perfused, but a pulseless, poorly perfused, mottled hand requires urgent reduction and fixation of the fracture. Incarceration of the brachial artery should be suspected if there is a block to reduction, and open reduction and retrieval of the anterior cubital structures from the site of the fracture are indicated. If reduction and fixation are achieved, but the hand remains poorly perfused, anterior exploration of the fracture and artery is indicated. Arterial spasm may improve after retrieval of the artery from the fracture. If the circulation is not then restored and the hand remains poorly perfused, vascular exploration and possible repair by an appropriate specialist are necessary. The question remains as to how to proceed if, after satisfactory reduction, the radial pulse does not return although the hand is warm and well-perfused, the so-called 'pink, pulseless hand'. The authors of two papers ${ }^{3,4}$ in this issue of the Journal consider this question further.

Blakey et $\mathrm{al}^{3}$ describe the outcome in 26 children referred to their institution at a mean of three months after fracture. The brachial artery was explored at this time in 21 children. In 12 the vessel was constricted by scar tissue and in nine it was trapped within the fracture. Interestingly, pulsatile flow returned in all 21 children after release or decompression. By contrast, Mangat et $\mathrm{al}^{4}$ describe 19 children presenting with a grade-III fracture who had been treated initially within a median time of six hours from injury and received one of two management strategies. A total of 11 were observed initially and the radial pulse returned in two within 24 hours, after three weeks in three and after one to three months in another two. Secondary exploration was undertaken in four children who had an absent pulse but well-perfused hands, and flow was restored in two instances. However, eight children had immediate exploration of the fracture and brachial artery and in all the radial pulse returned after three weeks. The return of a pulse does not necessarily indicate a patent brachial artery, ${ }^{4}$ and it is often assumed that the collateral circulation at the elbow may be sufficient to perfuse the hand in a child.

Anatomists recognise variations in the principal arteries of the upper limb, particularly the two main vessels traversing the antecubital fossa. These comprise a deep, or normal, brachial artery and a superficial brachial, radial or ulnar artery. ${ }^{5}$ Anastomoses between these two trunks have been reported as an incidental finding in $1 \%$ to $6 \% .^{5}$ The number of children reported in the literature who had an isolated loss of the brachial artery and no concomitant neurological deficit was very small. Lally et $\mathrm{al}^{6}$ reviewed 11 children about 15 years after ligation of a brachial artery for the insertion of a Scribner shunt. None had critical ischaemia of the limb at follow-up although several had mild claudication and a lower systolic pressure on the ligated side. Garbuz, Leitch and Wright ${ }^{7}$ described one patient with an open supracondylar fracture in whom the lacerated brachial artery was ligated. At follow-up she complained of pain in the wrist after continuous writing and had a diminished radial pulse. Lipscomb and Burleson ${ }^{8}$ resected the damaged segment of the brachial artery in eight children 
and reported a full functional recovery with no evidence of ischaemic contracture. It seems from these reports ${ }^{6-8}$ that loss of the brachial artery in a child is compatible with acceptable function, in the absence of a neurological injury.

The term pink, pulseless hand may convey the impression that this is solely a vascular problem. This is clearly not always the case. Out of 19 children in the series of Mangat et al, ${ }^{4}$ nine had a nerve palsy and in that of Blakey et $\mathrm{al}^{3}$ there were 56 nerve lesions in 26 children. The difference in the number of neurological lesions and functional outcome between the series may be because one report ${ }^{3}$ is from a tertiary referral centre. The incidence of neurological injury associated with a type III fracture without vascular compromise is approximately $10 \%$ to $20 \%$ and the anterior interosseous nerve is most commonly injured. ${ }^{1}$ Luria et $\mathrm{al}^{9}$ and Mangat et $\mathrm{al}^{4}$ found a correlation between the vascular injury and deficit of the median nerve, which is not surprising, given their anatomical proximity in the antecubital fossa. Garbuz et $\mathrm{al}^{7}$ found that in 22 children presenting with an absent radial pulse, 12 had an associated nerve injury, an incidence of $55 \%$. The neurological component of the pink, pulseless hand may result from ischaemia to the peripheral nerve, compression by swollen, infarcted muscle or as a result of direct trauma by being trapped in the fracture or tented over the distal humerus. Secondary damage to a peripheral nerve may result from manipulation of the fracture or hyperflexion of the swollen elbow as part of conservative management. The consequences to the circulation of flexing an already tense elbow have been described by Charnley ${ }^{10}$ as being similar to the kinking of a distended balloon. Fixation by Kirschner wires as part of the management of a Gartland type III fracture is an accepted treatment option but, interestingly, Blakey et $\mathrm{al}^{3}$ reported that five children referred to their unit were treated by manipulation only and had no fixation. The Gartland grade of the fractures was not given.

Orthopaedic surgeons involved in paediatric trauma are accustomed to seeing children who have a poor or absent radial pulse which returns after reduction of a supracondylar fracture. The pink, pulseless hand, with or without a neurological deficit, is very uncommon, or under-reported. The incidence is not established and the functional outcome in children who had a pink, pulseless hand, but in whom no intervention was undertaken, are also unknown in the population as a whole. Mangat et al ${ }^{4}$ collected 19 cases over 14 years and Blakey et $\mathrm{al}^{3} 26$ cases over 20 years. A recent survey of Pediatric Orthopaedic Society of North America members by White, Mehlman and Crawford ${ }^{11}$ reported this year in the Proceedings of the European Paediatric Orthopaedic Society, identified 102 cases of perfused, pink hands, 61 of which remained pulseless after closed reduction and stabilisation. Of the latter, $77 \%$ had a documented injury to the brachial artery. Patency rates after revascularisation appeared to be sufficiently high to justify intervention. By contrast, Sabharwal et $\mathrm{al}^{12}{ }^{12}$ in an earlier series, noted a high rate of asymptomatic occlusion and residual stenosis after repair. In this study the radial pulse was present in 11 of 13 children who were seen at follow-up after vascular exploration for a pink, pulseless hand. Additionally, all underwent colour-flow Duplex ultrasonography and ten had MR angiography. There was evidence of occlusion of the distal brachial artery with reconstitution of forearm flow through the collateral circulation in two children and three had residual stenosis of the brachial artery, with increased collateral circulation around the lesion giving good antegrade flow. The authors concluded that "It appears that had the vascular reconstruction not been done, collateral circulation would have been adequate to maintain a viable extremity". Vascular studies, apart from clinical examination, at follow-up were not recorded by Blakey et $\mathrm{al}^{3}$ or Mangat et al. ${ }^{4}$

Opinions differ as to whether or not the artery should be explored after reduction and fixation of the fracture when the hand remains well-perfused, but pulseless. Some authors ${ }^{2,7,13,14}$ have advocated a conservative approach. By contrast, Noaman ${ }^{15}$ explored the brachial artery in 31 children in whom the radial pulse was absent after closed reduction and pinning in a series of 840 grade-III fractures. His indications were a pulseless forearm with a pink or cold hand, an absent radial pulse one hour after satisfactory closed reduction and percutaneous pinning and an absent radial pulse associated with an open fracture or signs of tethering of brachialis. There was arterial damage in 30 children and in one the artery was released from the site of the fracture. Korompilias et $\mathrm{al}^{16}$ also recommended surgical exploration to restore the patency of the brachial artery, based on their experience of five children with a pink, pulseless hand. However, Griffin et $a l,{ }^{17}$ in their review of the literature of the management of vascular complications of supracondylar fractures, concluded that "a child with a pink pulseless hand following successful fracture reduction can be managed expectantly but if additional signs of ischaemia are present, surgical exploration is required".

Will these two papers ${ }^{3,4}$ change clinical management? Interested readers will decide for themselves. The number of pink, pulseless hands reported in the literature after reduction and stabilisation of a fracture is remarkably small, given the frequency of these injuries. Little is known of the natural history of the pink, pulseless hand in the population at large. The literature is not always clear in distinguishing between critical ischaemia and a pink, pulseless hand. Both papers ${ }^{3,4}$ highlight the fact that the pink, pulseless hand is not necessarily an isolated vascular problem and neurological damage is often a feature. The paper by Blakey et $\mathrm{al}^{3}$ reminds the reader of the consequences of prolonged ischaemia. Mangat et $\mathrm{al}^{4}$ advocate early exploration in patients with a co-existing palsy of the median or anterior interosseous nerves, but these nerves are the most commonly injured after a supracondylar fracture. Both papers ${ }^{3,4}$ recommend exploration of the brachial artery, but neither document the patency of the vessel, evaluated by 
vascular studies, at follow-up. The clinical outcome from these two papers ${ }^{3,4}$ is very different and the distinction between direct vascular and neurological damage and compartment syndrome, also a very unusual outcome after supracondylar fracture, ${ }^{18}$ may be a factor. There is no controversy as to the need to explore the brachial artery when the hand is white, pulseless and clearly ischaemic after reduction and stabilisation of the fracture. Until further information is available it would be reasonable to monitor carefully a pink, pulseless hand over the ensuing 24 to 48 hours after satisfactory reduction and pinning of the fracture. If perfusion of the hand deteriorates during this time, the pain worsens and there are signs of a deteriorating neurological picture, exploration of the brachial artery and the affected nerve(s) is indicated.

No benefits in any form have been received or will be received from a commercial party related directly or indirectly to the subject of this article.

\section{References}

1. Omir R, Choi PD, Skaggs DL. Supracondylar humeral fractures in children. J Bone Joint Surg [Am] 2008;90-A:1121-32.

2. Gartland JJ. Management of supracondylar fractures in the humerus in children. Surg Gynecol Obstet 1959;109:145-54.

3. Blakey CM, Biant LC, Birch R. Ischaemia and the pink, pulseless hand complicating supracondylar fractures of the humerus in childhood: long-term follow-up. J Bone Joint Surg [Br] 2009;91-B:1487-92

4. Mangat KS, Martin A, Bache CE. The 'pulseless pink' hand following supracondylar fractures of the humerus in children: the predictive value of nerve palsy. J Bone Joint Surg [Br] 2009;91-B:1521-5.
5. Rodriguez-Niedenführ M, Sanudo JR, Vázquez T, et al. Anastomosis at the level of the elbow joint connecting the deep, or normal, brachial artery with major arterial variations of the upper limb. J Anat 2000;196:115-19.

6. Lally KP, Foster CE 3rd, Chwals WP, Brennan LP, Atkinson JB. Long-term follow-up of brachial artery ligation in children. Ann Surg 1990;212:194-6.

7. Garbuz DS, Leitch K, Wright JG. The treatment of supracondylar fractures in children with an absent radial pulse. J Pediatr Orthop 1996;16:594-6.

8. Lipscomb PR, Burleson RJ. Vascular and neural complications in supracondylar fractures of the humerus in children. J Bone Joint Surg [Am] 1955;37-A:487-92.

9. Luria S, Sucar A, Eylond S, et al. Vascular complications of supracondylar humeral fractures. J Pediatr Orthop B 2007;16:133-43.

10. Charnley J. Supracondylar fractures of the humerus in children. In: 'The closed treatment of common fractures'. Fourth ed. Colt Books, Cambridge 1999:113.

11. White L, MehIman CT, Crawford AH. Pulseless and puzzling, vascular injuries in supracondylar humeral fractures in children: a meta analysis of observational studies and results of a POSNA membership survey. J Child Orthop 2009;3(Suppl 1):12.

12. Sabharwal S, Tredwell SJ, Beauchamp RD, et al. Management of pulseless pink hand in pediatric supracondylar fractures of the humerus. J Pediatr Orthop 1997;17:303-10.

13. Gosens T, Bongers KJ. Neurovascular and functional outcome in displaced supracondylar fractures of the humerus in children. Injury 2003;34:267-73.

14. Louahem DJ, Nebunescu A, Canavese F, Dimeglio A. Neurovascular complications and severe displacement in supracondylar humeral fractures in children: defensive or offensive strategy. J Pediatr Orthop B 2006;15:51-7.

15. Noaman HH. Microsurgical reconstruction of brachial artery injuries in displaced supracondylar fracture humerus in children. Microsurgery 2006;26:498-505.

16. Korompilias AV, Lykissas MG, Mitsionis GI, et al. Treatment of pink pulseless hand following supracondylar fractures of the humerus in children. Int Orthop 2009;33:237-41.

17. Griffin KJ, Walsh SR, Markar S, et al. The pink pulseless hand: a review of the literature regarding management of vascular complications of supracondylar humeral fractures in children. Eur J Vasc Endovasc Surg 2008;36:697-702.

18. Ramachandran M, Skaggs DL, Crawford HA, et al. Delaying treatment of supracondylar fractures in children: has the pendulum swung too far? J Bone Joint Surg [Br] 2008;90-B:1228-33 